\title{
Testing Win X-ray, a Monte Carlo Program for X-ray Microanalysis in the Scanning Electron Microscope
}

\author{
Helen Campbell,* and Raynald Gauvin* \\ * Department of Mining, Metals \& Materials Engineering, McGill University, M.H.Wong \\ Building, 3610 University Street, Montreal, Quebec, Canada, H3A 2B2
}

Monte Carlo programs for simulating the behavior and effects of beam electrons in a scanning electron microscope (SEM) are well known. "Casino" [1], released in 1997 by Hovington, Drouin and Gauvin, is one of these programs, and it was followed in 2003 by "Win X-ray" (available free of charge at www.montecarlomodeling.mcgill.ca) [2]. Win $\mathrm{X}$-ray was developed to compute the full x-ray spectrum (characteristic lines plus the bremmstrahlung) that would be measured with an energy dispersive spectrometer (EDS) system in the SEM. It can be applied to homogeneous alloys and to compounds.

It is the first step towards producing a Monte Carlo program for quantitative microanalysis in the SEM, but before this can be developed it is essential to evaluate the program by comparing the spectra it produces with real spectra obtained in the SEM. In this paper, some of these comparisons are presented.

A JEOL 840A scanning electron microscope with an EDAX Phoenix system was used to produce the experimental spectra. The "Sapphire" x-ray detector has a "super ultra thin" window to permit the detection of low-energy x-rays. The time-constant used was $100 \mu$ secs. The take-off angle was $30^{\circ}$ with a Working Distance of $15 \mathrm{~mm}$. The results shown are the average of 3 acquired spectra for each of which the live-time of acquisition was 100 secs.

The specimens used were x-ray microanalysis standards from NIST that had been suitably mounted and polished. Attached are the results for two of these standards - an alloy consisting of $60 \% \mathrm{Au}$ with $40 \% \mathrm{Cu}$, and $\mathrm{TiC}$.

Figs. 1 and 2 show the results for the $\mathrm{Au}-\mathrm{Cu}$ alloy and the $\mathrm{TiC}$ respectively. There is good agreement in the case of the $\mathrm{Au}-\mathrm{Cu}$. However, for the $\mathrm{TiC}$ below approximately $2 \mathrm{keV}$, the computed values are higher than the experimental values, probably because the bremmmstrahlung cross-sections in the model are too high for low x-ray photon energy.

\section{References}

[1] P. Hovington, D. Drouin and R. Gauvin (1997), "Casino: A New Era of Monte Carlo Code in C Language for Electron Beam Interaction, Part I: Description of the Program”, Scanning, Vol.19, pp.1-14

[2] R. Gauvin, E. Lifshin, H. Demers, P. Horny and H. Campbell (2003), "Win X-ray, a new Monte Carlo Program that Computes X-ray Spectra for X-ray Microanalysis in the Scanning Electron Microscope", submitted to Microscopy and Microanalysis. 


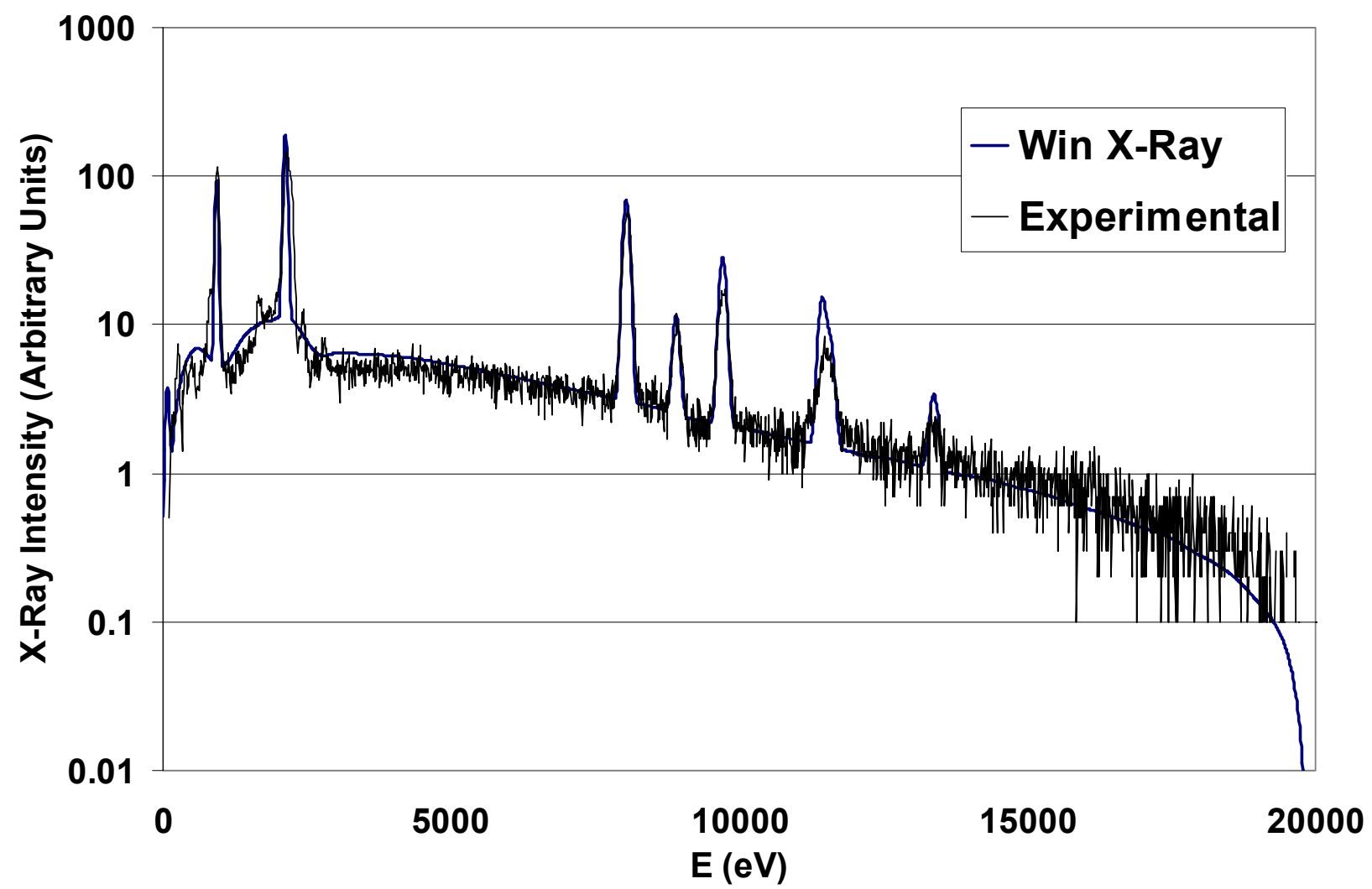

Fig.1. Experimental and computed spectra for $60 \% \mathrm{Au}-40 \% \mathrm{Cu}$ alloy at $20 \mathrm{keV}$.

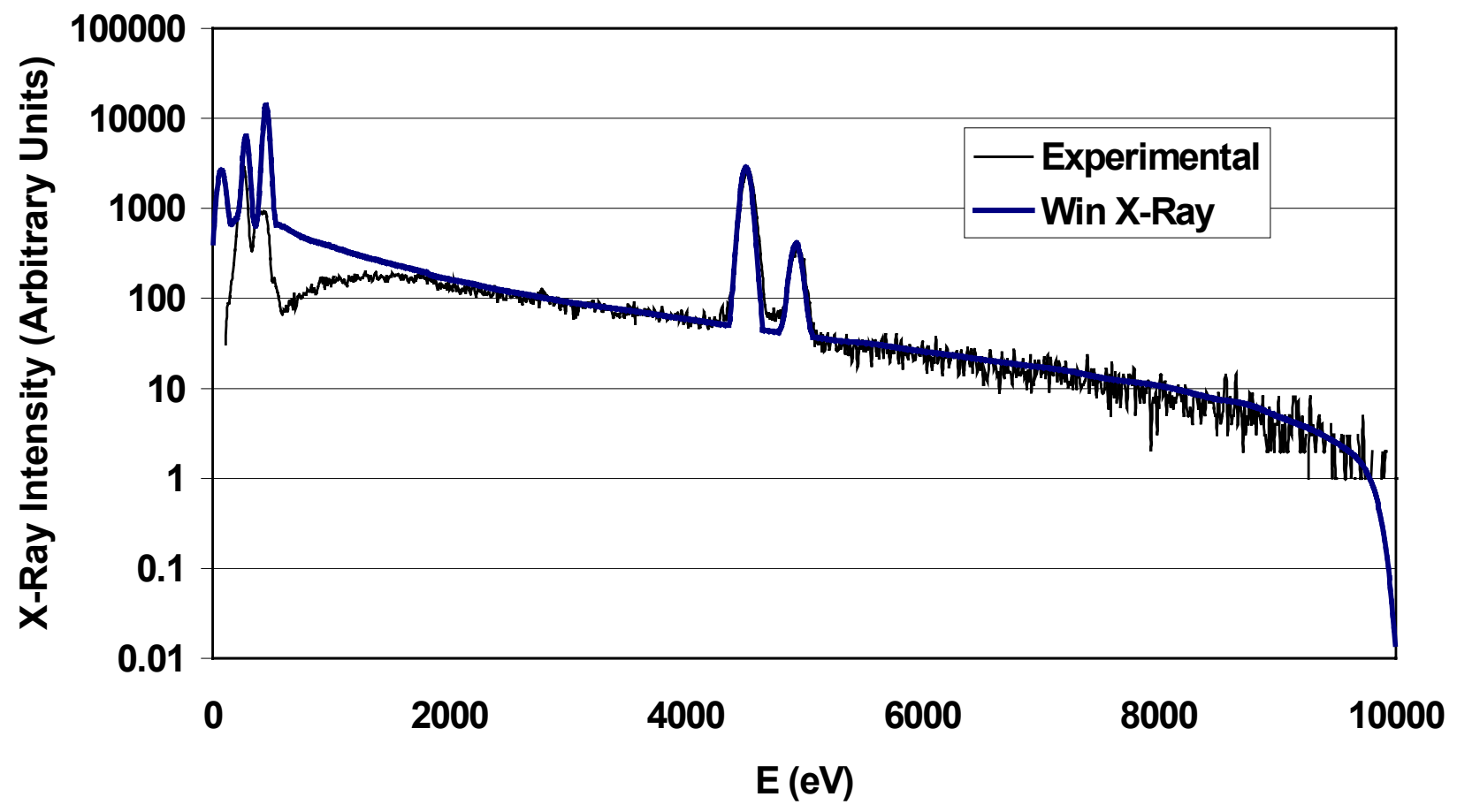

Fig.2. Experimental and computed spectra for $\mathrm{TiC}$ at $10 \mathrm{keV}$. 